\title{
Early Detection of Metastases by Bone Scintigraphy in Patients with Hepatocellular Carcinoma
}

\author{
Xiaohui Wang ${ }^{1}$, Jiangyan $\mathrm{Liu}^{1}$, Gang $\mathrm{Chen}^{2}, \mathrm{Yu} \mathrm{Li}^{2}$ and $\mathrm{Yumin}_{\mathrm{Li}^{2}} 3^{*}$ \\ ${ }^{1}$ Department of Nuclear Medicine, Lanzhou University Second Hospital, Lanzhou, China \\ ${ }^{2}$ First Department of General Surgery, Lanzhou University Second Hospital, Lanzhou, China \\ ${ }^{3}$ Gansu Provincial Key Laboratory of Digestive System Tumors, Lanzhou University Second Hospital, Lanzhou, China
}

"Corresponding author: Yumin Li, Gansu Provincial Key Laboratory of Digestive System Tumors; First Department of General Surgery, Lanzhou University Second Hospital, Lanzhou, Gansu Province, 730030, China, Tel: +8613893615421; Email: liym@lzu.edu.cn

Rec date: November 18, 2015; Acc date: January 09, 2016; Pub date: January 18, 2016

Copyright: (c) 2016 Wang X, et al. This is an open-access article distributed under the terms of the Creative Commons Attribution License, which permits unrestricted use, distribution, and reproduction in any medium, provided the original author and source are credited.

Abstract
Hepatocellular carcinoma (HCC) is relatively common worldwide. Until a few years ago skeletal involvement from
HCC was rarely diagnosed. Thanks to novel imaging techniques and multidisciplinary treatment approaches, the
overall survival in HCC patients has prolonged, and bone involvement has significantly increased currently. This
short review summaries the value of bone scintigraphy in patients with hepatocellular carcinoma.

Keywords: Hepatocellular carcinoma; Bone metastases; Single photon emission computed tomography

\section{Introduction}

Although bone involvement is reported as uncommon in hepatocellular carcinoma (HCC), its incidence has significantly increased in the last decade due to novel imaging techniques and multidisciplinary treatment approaches and the overall survival in HCC patients has prolonged [1].

Bone metastases were most frequently found in the pelvis (20\%), followed by the lumbar spine (14\%) and long bones (13\%) [2], and bone metastasis is discovered as the first symptoms in about $5 \%-7 \%$ of the HCC patients, which would cause clinical misdiagnosis easily. We ever reported a patient with a half year history of right hip pain, who was diagnosed as primary femoral benign tumor initially. A single photon emission computed tomography (SPECT) of total skeletal bones showed abnormal radioactive tracer uptake widely in acetabulum, femoral head, neck of femur, large rotor and the third right frontal rib (Figure 1), while CT scan only showed lesions in the right side of the femoral head and the large rotor) [3].

Bone scintigraphy (BS) can be an option for patients to reduce the misdiagnosis rate and to rule out the possibility of bone metastasis from HCC, which could give a comprehensive assessment to whole body bones. 99mTc-MDP (Technetium-99m-methylene diphosphonate) is usually used as imaging agent. The kidneys, bladder and sometimes the ureter can be seen in the imaging, due to that the imaging tracer is typically excreted by urinary system. Hence the right renal abnormality (reached the pelvis and with irregular shape) was detected by bone scintigraphy in the reported case (Figure 1). Subsequently, non-enhanced and then contrast-enhanced CT of abdomen was taken, which demonstrated a huge enhanced mass in the right lobe of the liver (showed increased contrast enhancement within the lesion during the arterial phase). The patient was given right hip joint replacement, and the pathology diagnosis was liver metastasis carcinoma [3].

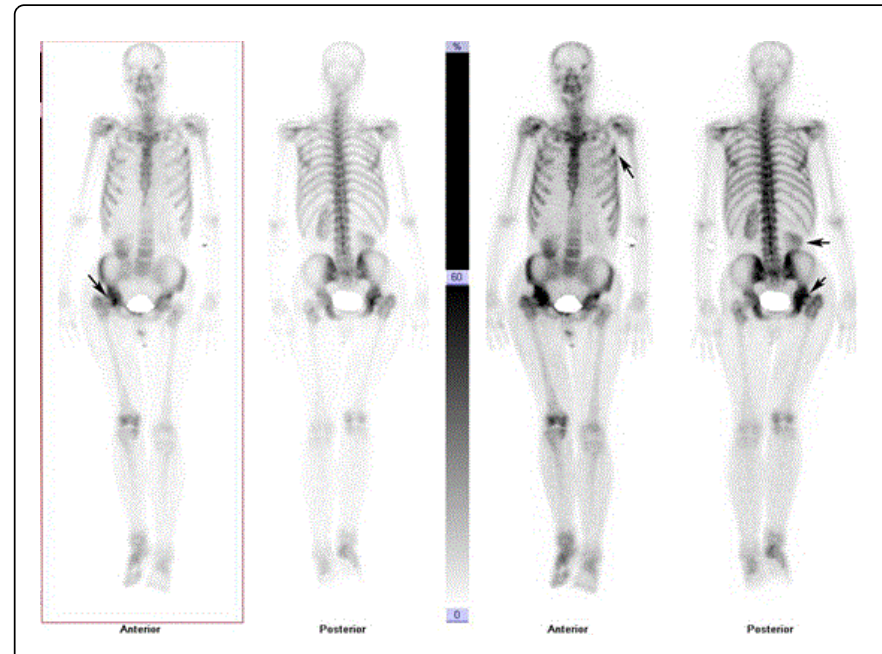

Figure 1: (Hip joint and femur metastases as the first symptom of hepatocellular carcinoma detected by bone scintigraphy. 2015 . Radiology of Infectious Diseases.)

It is generally accepted that bone scintigraphy is often more sensitive than structural imaging, which can detect lesions earlier 3-6 months than CT. Yang et al. Ever compared 18F-FDG PET, CT, MRI and $\mathrm{BS}$ in diagnosis of bone metastases, which indicated that the pooled sensitivity estimates for PET, CT, MRI and BS were: PET = MRI $>\mathrm{BS}(90.4 \%)>\mathrm{CT}(75.1 \%)$ and the pooled specificity estimates for PET, $\mathrm{CT}, \mathrm{MRI}$ and $\mathrm{BS}$ were: $\mathrm{PET}>\mathrm{MRI}>\mathrm{BS}(93.6 \%)>\mathrm{CT}(83.2 \%)$ [4].

Bone metastases in hepatocellular carcinoma has been becoming an emerging issue [1]. Bone scintigraphy may potentially be an option for patients to reduce the misdiagnosis rate and to rule out the possibility of bone metastasis from HCC. Furthermore, it was able to present additional information concerning kidneys, to which more attention should be paid. 
Citation: Wang X, Liu J, Chen J, Li Y, Li Y (2016) Early Detection of Metastases by Bone Scintigraphy in Patients with Hepatocellular Carcinoma. J Liver 5: 191. doi:10.4172/2167-0889.1000191

Page 2 of 2

\section{References}

1. Longo V, Brunetti O, D'Oronzo S, Ostuni C, Gatti P, et al. (2014) Bone metastases in hepatocellular carcinoma: an emerging issue. Cancer Metastasis Rev 33: 333-342.

2. Seo HJ, Kim GM, Kim JH, Kang WJ, Choi HJ (2015) 18F-FDG PET/CT in hepatocellular carcinoma:detection of bone metastasis and prediction of prognosis. Nucl Med Commun 36: 226-233.
3. Wang X, Liu J (2015) Hip joint and femur metastases as the first symptom of hepatocellular carcinoma detected by bone scintigraphy. Radiology of Infectious Diseases 2: 134-136.

4. Yang HL, Liu T, Wang XM, Xu Y, Deng SM (2011) Diagnosis of bone metastases: a meta-analysis comparing 18F-FDG PET, CT, MRI and bone scintigraphy. Eur Radiol 21: 2604-2617. 